\title{
Atypical Pancreatic Cystadenocarcinoma in Pregnant Women: A Case Report
}

\author{
Han $\mathrm{Li}^{1}$, Dongdong $\mathrm{Ji}^{1}$, Chenglong Guo ${ }^{2}$, Xuewen Wang ${ }^{1}$, Xingyuan Zhang ${ }^{1}$, Lingqun Kong ${ }^{1, *}$ \\ ${ }^{1}$ Department of Hepatobiliary Surgery, Binzhou Medical University Hospital, Binzhou City, People's Republic of China \\ ${ }^{2}$ Department of Health examination, Binzhou Medical University Hospital, Binzhou City, People's Republic of China
}

\section{Email address:}

Mimihalu995@163.com (Han Li),634717302@qq.com (Dongdong Ji), 1058200255@qq.com (Chenglong Guo), wangxuewen1993@163.com (Xuewen Wang), 1058200255@qq.com (Xingyuan Zhang), gdwk52933257@163.com (Lingqun Kong)

${ }^{*}$ Corresponding author

\section{To cite this article:}

Han Li, Dongdong Ji, Chenglong Guo, Xuewen Wang, Xingyuan Zhang, Lingqun Kong. Atypical Pancreatic Cystadenocarcinoma in Pregnant Women: A Case Report. American Journal of Internal Medicine. Vol. 9, No. 4, 2021, pp. 194-197. doi: 10.11648/j.ajim.20210904.15

Received: July 6, 2021; Accepted: July 19, 2021; Published: July 29, 2021

\begin{abstract}
Pancreatic cystic lesion (PCL) is a relatively low incidence of pancreatic disease. No clinical symptoms of PCL prevalence rate is $2.4 \% \sim 13.5 \%$ and has a tendency to increase with the increase of age. With the development of modern imaging technology and the improvement of people health consciousness, there has been a dramatic increase in the prevalence of PCL. PCLs involving a wide range of pathology can range from obviously benign to borderline malignant potential lesions to overt malignancy. Pancreatic cystadenocarcinoma belongs to one of the malignant tumor of PCLs, may be malignant from pancreatic cystadenoma, and it is clinically rare, accounting for only $1 \%$ of the malignant tumors of the pancreas. Preoperative diagnosis is difficult of the disease, it mostly need to postoperative pathological diagnosis. The main symptoms of pancreatic cystadenocarcinoma are dull pain or low back pain in the upper and middle abdomen, and mass in the upper abdomen. Abdominal pain is not intense, some patients only for the feeling of fullness discomfort. Other symptoms may include loss of appetite, nausea, indigestion, weight loss, jaundice, and in a few patients, gastrointestinal bleeding. In this paper, we report a rare case of pancreatic cystadenocarcinoma with a huge cystic lesion that grows rapidly in a short period of time in pregnant women, which preoperative diagnosis is considered pancreatic cystadenoma in the preoperative. Complete resection was performed and histological examination confirmed the diagnosis of pancreatic cystadenocarcinoma.
\end{abstract}

Keywords: Pancreatic Cystic Lesion, Pancreatic Cystadenocarcinoma, Pancreatic Cystadenoma,

Pancreatic Cystic Neoplasm

\section{Introduction}

Pancreatic cystic lesion (PCL) is a relatively uncommon condition. It is a neoplastic or nonneoplastic (single or multiple tumour-like) cystic lesion formed by pancreatic epithelial and/or stromal tissue, mainly including pancreatic cystic neoplasm (PCN) and pancreatic pseudocyst (PP). According to the 2010 WHO classification criteria, PCN mainly includes serous cystic neoplasm (SCN), mucinous cystic neoplasm (MCN), intraductal papillary mucinous neoplasm (IPMN) and solid pseudopapillaryneoplasm (SPN), etc. [1, 2] Due to the differences in clinical characteristics, imaging manifestations and pathological features of PCLs, it is still a big problem and challenge to improve the accuracy of preoperative diagnosis. In the meantime, due to the different types of lesions between the potential risk of malignant transformation, there are huge differences in the corresponding treatment, follow-up strategy and clinical prognosis.

\section{Case Presentation}

The patient, a 31-year-old female was admitted to our hospital with 1-month history of intermittent abdominal pain. She had a history of Pancreatic Cyst for 6 month and 16 weeks of pregnancy. Six months ago her regular physical examination in our hospital medical center of the chest computed tomography $(\mathrm{CT})$ revealed the pancreatic tail class elliptic low density shadow, the largest cross-sectional area 
were about $4.2 \mathrm{~cm} \times 3.7 \mathrm{~cm}$, and the boundary is clear. (Figure 1 A) Base on the above conditions, the diagnosis of PCL (pancreatic cystadenoma?) should be considered. Since the patient is a young woman with no symptoms and combined with the patient's wishes, regular examinations should be recommended. After two months, she became pregnant and did not check again. Nonetheless, after another four months, she appeared left upper abdominal pain a month ago and then in our hospital again. Physical examination revealed tenderness of upper abdominal mass. Her biochemical indicators (liver function, renal function, etc.) were within normal ranges. But tumor marker results were as abnormal: alpha-fetoprotein (AFP), 19.47ng/ml (normal range, 0-13.6), carbohydrate antigen 19-9 (CA19-9), 45.19U/ml (normal range, 0-27), and carcinoembryonic antigen (CEA) was normal. Magnetic Resonance Imaging (MRI) revealed huge long T1 and long T2 signals and its internal signal evenly in the pancreatic tail, without obvious enhancement scanning reinforcement. The size of the lesion is about $10.6 \mathrm{~cm} \mathrm{x} 10.8$ $\mathrm{cm} \times 15.7 \mathrm{~cm}$ and the boundary is clear. (Figure $1 \mathrm{~B}$ and $1 \mathrm{C}$ ) A huge pancreas cystic tumor was diagnosed and was suspected possibility for benign tumors: pancreatic cystadenoma? But the cyst accompanied by obvious symptoms was rapid increased in a short period of time and operative exploration was undertaken. About $16 \mathrm{~cm}$ in diameter cystic tumor was found in the pancreatic tail and its texture was firm. Empty needle puncture pulled about $900 \mathrm{ml}$ light yellow clear liquid. To further explore the mass originated in pancreatic tail, base pancreatic tissue and pancreatic head groups are a hard.
Around the pancreas and mesenteric root has no enlarged lymph node. Three rapid pathologic examinations during the operation finally revealed hyperplasia of dysplastic glands in the pancreatic tissue supporting malignancy. Radical distal pancreatectomy combined with splenectomy was performed and the tumour was completely removed. Histologic examination showed the fibrous capsule wall has no epithelial lining cover and partial section was hemorrhage, acute or chronic inflammatory cells were infiltration at the same time. The basal parts of tumor was found Medium - high differentiation of the adenocarcinoma and its cut edge adjacent to the pancreas. (Figure 2 A-C) Immunohistochemistry showed positive expression of Cytokeratin (CK), CK7 and CEA, whereas expression of P53 was negative. In addition, a mitotic rate about $15 \%$ staining for proliferative marker Ki-67 was detected. (Figure 3 A-E) The tumor marker test results of liquid in tumor were as follow: CA19-9, >1000U/ml (normal range, 0-27), and CEA, $19.58 \mathrm{ng} / \mathrm{ml}$ (normal range, 0-3.4), hormone levels in tumor were also abnormal: estradiol, $400.70 \mathrm{pmol} / \mathrm{L}$, progesterone $\mathrm{p}$, $4.490 \mathrm{nmol} / \mathrm{L}$, testosterone, $0.91 \mathrm{nmol} / \mathrm{L}$. The diagnosis of pancreatic cystadenocarcinoma was confirmed. The patient refused any adjuvant chemotherapy after radical distal pancreatectomy combined with splenectomy. The patient recovered uneventfully and was discharged after the operation. MRI examination of the abdomen after one and a half months suggested a recurrence of the pancreatic cystadenocarcinoma and follow-up was lost".
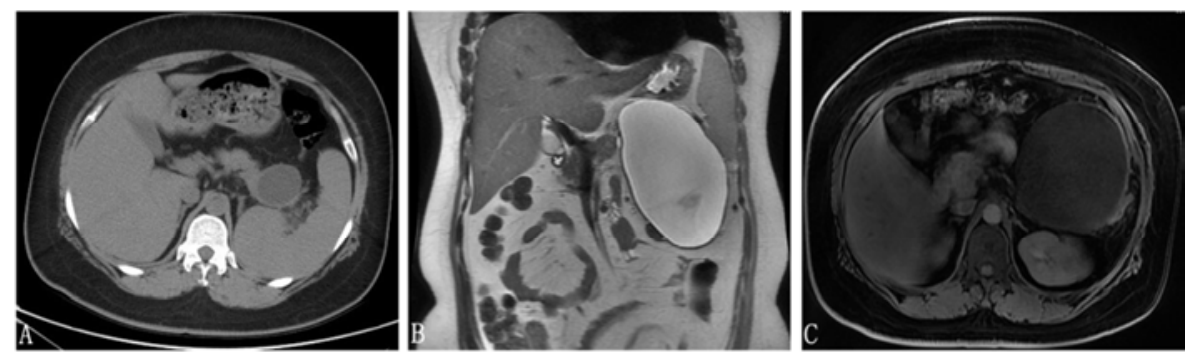

Figure 1. A. The chest computed tomography B C. Magnetic Resonance Imaging
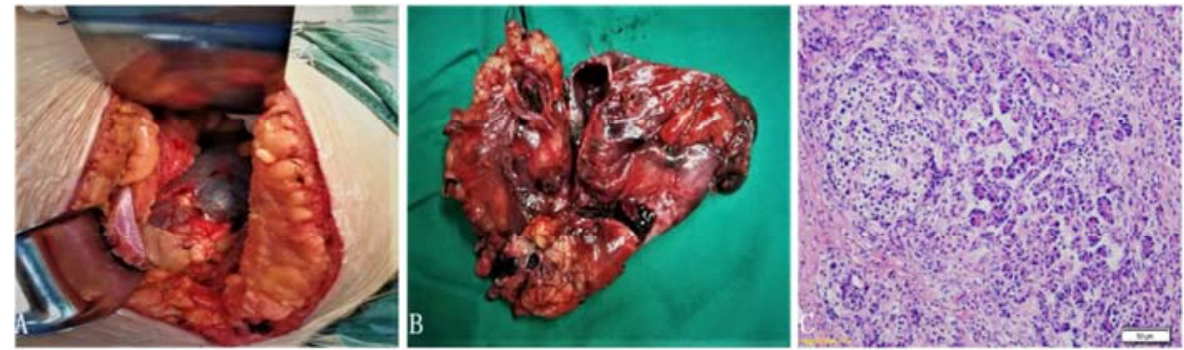

Figure 2. A B Tumor tissue C Immunohistochemistry.

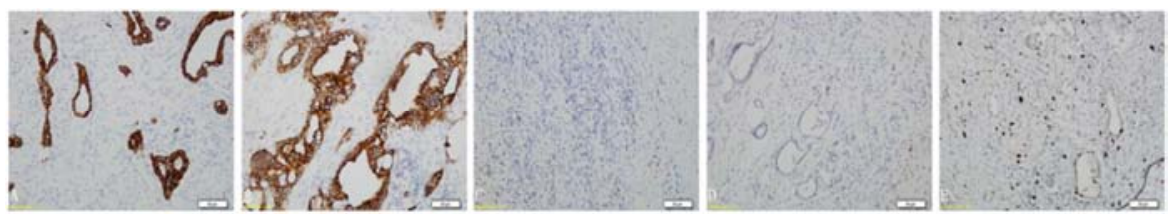

Figure 3. The tumor marker test results of liquid in tumor. 


\section{Discussion}

PCL is a relatively uncommon condition, but there has been a dramatic increase in the prevalence recently because of the widespread use of high-resolution imaging, as well as the aging of the population. Although cystic lesions of the pancreas are relatively rare, they are considered to be an increasingly important category with a challenging differential diagnosis. Pancreatic cystadenocarcinoma belongs to one of the malignant tumor of PCLs, may be malignant from pancreatic cystadenoma, and it is clinically rare, accounting for only $1 \%$ of the malignant tumors of the pancreas. [3] Due to the hidden and non-specific clinical manifestations and laboratory examination indicators, it is generally difficult to diagnose the disease, and is easily confused with pancreatic cystadenoma and PP, resulting in misdiagnosis and misdiagnosis, most of which can only be confirmed by pathological biopsy.

Pancreatic cystadenoma and Pancreatic cystadenocarcinoma are originated in the duct system and belong to the proliferative cyst. Combined with clinical differential diagnosis, pancreatic cystic tumor has been divided into two categories: one is SCN, which is divided into serous cystadenoma and serous cystadenocarcinoma, and the other is $\mathrm{MCN}$, which is more rare than $\mathrm{SCN}$, which is divided into mucinous cystadenoma and mucinous cystadenocarcinoma of the pancreas. SCN accounts for $16 \%$ of PCLs, the vast majority of which are asymptomatic benign lesions with a good prognosis, and is common in women over the age of $50(75 \%)$. [4] The study found that the risk of SCN malignant transformation into serous cystadenocarcinoma was very low $(0.1 \%)$. [5] The European evidence-based guidelines recommend that patients diagnosed with SCN radiologically without clinical symptoms should be followed up for 1 year, and a follow-up plan based on the patient's symptoms should be established after 1 year. [6] MCN is mainly found in middle-aged women, usually located in the tail of the pancreatic body, with malignant potential. [7] Mucinous cystadenocarcinoma of the pancreas is structurally similar to mucinous cystadenoma, but the volume is generally larger than the latter. Meanwhile, Cystic cavity of mucinous cystadenocarcinoma has obvious papillae structure and imaging examination can see its interior separation and papillary structure. The European evidence-based guidelines recommend surgical treatment for tumors up to $4 \mathrm{~cm}$ in diameter and for MCNs with symptoms or risk factors (such as mural nodules), regardless of size; For $\mathrm{MCN}$ with tumor diameter $<4 \mathrm{~cm}$ and no risk factors, follow-up was safe. [6]

Imaging examination is a major means of diagnosis of PCLs. MRI or magnetic resonance cholangiopancreatography (MRCP) has been reported to be between $40 \%$ and $95 \%$ accurate in identifying specific types of PCLs, while enhanced CT has been reported to be between $40 \%$ and $81 \%$ accurate. [8-12] Considering MRI's safety (no radiation) and higher diagnostic accuracy, the guidelines recommend MRI as the preferred method of examination. $[6,13]$ In recent years, with the development of the endoscopic ultrasonography (EUS) technology, not only can clearly show the pancreas cystic, oven and tissue around the lesion of pancreatic parenchyma and the pancreatic duct also have very good resolution, more fine endoscopic ultrasonography -guided fine-needle aspiration (EUS-FNA) can obtain tissue and fluid, now has become one of the commonly diagnostic method of such lesions. The capsule fluid obtained by EUS-FNA can be examined for amylase CEA and CA19-9 level and cytology, similar to MRI and CT, but not recommended as a single assessment tool due to its more invasive nature. [14]

The patients in this case has been found that pancreatic cystic for half a year ago and considerd pancreatic benign tumors by imaging examination. This did not cause her enough attention, and she also did no further relevant inspection to be clear diagnosis. Within half a year, the tumor from the diameter of about $4 \mathrm{~cm}$ rapid growth to $16 \mathrm{~cm}$ in diam. Combined with patient history, examination and intraoperative and postoperative pathologic results, we need reflection is mainly composed of the following: firstly, patients with preoperative CT and MRI are prompt cystic lesion, consider a benign, even in the short term increased rapidly, the density of cysts or signal and border no abnormal change, hints for pancreatic cystic placeholder cannot just rely on imaging diagnosis. Secondly, during pregnancy, this patient with progressive enlargement of the pancreas tumor has apparently unusual sex hormone levels of intra-operative puncture fluid. It further confirmed that pancreatic cancer as a hormone reactive tumor is closely associated with hormone levels. Lastly, it can be used as a risk factor for pregnancy.

\section{Conclusion}

Patients with cystic disease of the pancreas, especially pregnant women, more attention should be put on the basis of imaging examination to further improve laboratory examination indicators (such as oncology, etc.) as a result, at the same time when it is necessary to give EUS-FNA guidance line extraction fluid CA19-9, CEA detection such as diagnosis, there are also reported the contents of a cyst CA15-3 protein expression can differentiate benign and malignant pancreatic cystic tumor. [2, 3] It needs surgical removal of the treatment necessary. Pancreatic cystadenocarcinoma is an aggressive tumor that is prone to recurrence and metastasis after surgical resection. Although chemotherapy was not effective for patients with advanced disease, operative resection followed by chemoradiation therapy seemed to be the most effective therapy.

\section{Abbreviations}

$\mathrm{PCL}=$ pancreatic cystic lesion, MRI=Magnetic Resonance Imaging, $\mathrm{CT}=$ computed tomography, $\mathrm{CK}=$ cytokeratin, EUS-FNA=endoscopic ultrasonography-guided fine-needle 
aspiration, $\mathrm{PCN}=$ pancreatic cystic neoplasm, $\mathrm{PP}=$ pancreatic pseudocyst, $\mathrm{SCN}=$ serous cystic neoplasm, $\mathrm{MCN}=$ mucinous cystic neoplasm, IPMN=intraductal papillary mucinous neoplasm, $\quad \mathrm{SPN}=$ solid pseudopapillaryneoplasm, AFP=alpha-fetoprotein, CA19-9=carbohydrate antigen19-9, $\mathrm{CEA}=$ carcinoembryonic antigen, $\mathrm{MRCP}=$ magnetic resonance cholangiopancreatography

\section{Author Contributions}

Han Li and Dongdong Ji contributed to literature research and draft the manuscript. Chenglong Guo and Xuewen Wang contributed to collect clinical data. Xingyuan Zhang contributed to provide the relevant images. Lingqun Kong contributed to corrections to the manuscript and figure editing. All authors have approved the final version of the manuscript.

\section{Statement}

Informed consent has been obtained in writing from the patients and is available for review by the editor.

\section{References}

[1] Bai XL, Zhang Q, Masood N, et al. Pancreatic cystic neoplasms: a review of preoperative diagnosis and management. J Zhejiang Univ Sci B, 2013, 14 (3): 185-194.

[2] Reichermeier KM, Caselitz M, Wagner S. An unusual case of a pancreatic cyst. Gastroenterology. 2014; 147: e1-2.

[3] Hackert T, Büchler MW. Cystic tumors of the pancreas. Chirurg. 2017; 88 (11): 903-904.

[4] Chu LC, Singhi AD, Haroun RR, et al. The many faces of pancreatic serous cystadenoma: Radiologic and pathologic correlation [J]. Diagn Interv Imaging, 2017, 98 (3): 191-202.
[5] Jais B, Rebours V, Malleo G, et al. Serous cystic neoplasm of the pancreas: a multinational study of 2622 patients under the auspices of the international association of pancreatology and european pancreatic club (european study group on cystic tumors of the pancreas). Gut, 2016, 65 (2): 305-312.

[6] European Study Group on Cystic Tumours of the Pancreas. European evidence-based guidelines on pancreatic cystic neoplasms. Gut, 2018, 67 (5): 789-804.

[7] Doulamis IP, Mylonas KS, Kalfountzos CE, et al. Pancreatic mucinous cystadenocarcinoma: Epidemiology and outcomes. Int J Surg, 2016, 35: 76-82.

[8] Sainani NI, Saokar A, Deshpande V, et al. Comparative performance of MDCT and MRI with MR cholangiopancreatography in characterizing small pancreatic cysts. Am J Roentgenol, 2009, 193 (3): 722-731.

[9] Visser BC, Muthusamy VR, Yeh BM, et al. Diagnostic evaluation of cystic pancreatic lesions. HPB (Oxford), 2008, 10 (1): 63-69.

[10] Song SJ, Lee JM, Kim YJ, et al. Differentiation of intraductal papillary mucinous neoplasms from other pancreatic cystic masses: comparison of multirow-detector $\mathrm{CT}$ and $\mathrm{MR}$ imaging using ROC analysis. J Magn Reson Imaging, 2007, 26 (1): 86-93.

[11] Jang DK, Song BJ, Ryu JK, et al. Preoperative diagnosis of pancreatic cystic lesions: the accuracy of endoscopic ultrasound and cross-sectional imaging. Pancreas, 2015, 44 (8): 1329-1333.

[12] Lee HJ, Kim MJ, Choi JY, et al. Relative accuracy of CT and MRI in the differentiation of benign from malignant pancreatic cystic lesions. Clin Radiol, 2011, 66 (4): 315-321.

[13] Elta GH, Enestvedt BK, Sauer BG, et al. ACG clinical guideline: diagnosis and management of pancreatic cysts. Am J Gastroenterol, 2018, 113 (4): 464-479.

[14] Tirkes T, Aisen AM, Cramer HM, et al. Cystic neoplasms of the pancreas; findings on magnetic resonance imaging with pathological, surgical, and clinical correlation. Abdom Imaging, 2014, 39 (5): 1088-1101. 